\title{
A FRAMEWORK FOR ROBUSTNESS ANALYSIS OF ROAD NETWORKS FOR SHORT TERM VARIATIONS IN SUPPLY
}

\author{
M.Snelder ${ }^{\mathrm{a}, \mathrm{b}, \mathrm{c}}$ (corresponding author), H.J. van Zuylen ${ }^{\mathrm{b}, \mathrm{c}, \mathrm{e}}$, L.H. Immers ${ }^{\mathrm{c}, \mathrm{d}}$ \\ E-Mail: maaike.snelder@tno.nl, h.j.j.vanzuylen@tudelft.nl, $\underline{\text { b.immers@ } @ \text { rstrail.nl }}$
}

${ }^{a}$ TNO, Van Mourik Broekmanweg 6, P.O. Box 49, 2600 AA Delft, The Netherlands

Tel.: +31 1526968 27, Fax: +31 152696854

${ }^{\mathrm{b}}$ Delft University of Technology, Faculty of Civil Engineering and Geosciences, Department of Transport and Planning, P.O. Box 5048, 2600 GA Delft, The Netherlands,

${ }^{\mathrm{c}}$ TRAIL research school

${ }^{\mathrm{d}}$ Catholic University of Leuven, CIB, Traffic \& Infrastructure, Celestijnenlaan 300A B-3001

Belgium

${ }^{\mathrm{e}}$ Hunan University, faculty Civil Engineering, Changsha, P.R. China

\begin{abstract}
There is a growing awareness that road networks, are becoming more and more vulnerable to unforeseen disturbances like incidents and that measures need to be taken in order to make road networks more robust. In order to do this the following questions need to be addressed: How is robustness defined? Against which disturbances should the network be made robust? Which factors determine the robustness of a road network? What is the relationship between robustness, travel times and travel time reliability? Which indicators can be used to quantify robustness? How can these indicators be computed? This paper addresses these questions by developing a consistent framework for robustness in which a definition, terms related to robustness, indicators and an evaluation method are included. By doing this, policy makers and transportation analyst are offered a framework to discuss issues that are related to road network robustness and vulnerability which goes beyond the disconnected definitions, indicators and evaluation methods used so far in literature. Furthermore, the evaluation method that is presented for evaluating the robustness of the road network against short term variations in supply (like incidents) contributes to the problem of designing robust road networks because it has a relatively short computation time and it takes spillback effects and alternative routes into account.
\end{abstract}

Keywords: robustness; vulnerability; reliability; definitions; indicators; evaluation method. 


\section{INTRODUCTION}

There is a growing awareness that road networks, are becoming more and more vulnerable to unforeseen disturbances like incidents because of the fact that the level of congestion keeps growing. Furthermore, it becomes more difficult to recover from unforeseen disturbances since the spare capacity in the network reduces both in place and in time. The opportunity costs of vulnerability in the Netherlands can for example increase to more than 4000 million euro per year in 2030 (Snelder et al., 2008). This raises the question which measures can be taken to reduce the vulnerability or to increase the robustness of the road network and where and when these measures should be applied. Before these questions can be answered first some other questions need to be addressed: How is robustness defined? Against which disturbances should the network be made robust? Which factors determine the robustness of a road network? What is the relationship between robustness, travel times and travel time reliability? Which indicators can be used to quantify robustness? How can these indicators be computed? These latter questions are the questions that are addressed in this paper. In Figure 1 the different steps that have to be followed to make a robust road network design are summarized. This paper focuses on the first four steps of Figure 1 (from context to evaluation method). The last three steps are however kept in mind, since the results of the first four steps should be applicable in network design.

$<$ Figure 1 about here>

The robustness of transportation networks is a relatively new research area. There are several definitions, but none of these are commonly accepted. Specific indicators for robustness are scarce and robustness against short-term uncertainties in supply and demand is, as far as is known to the authors, not yet explicitly considered in the network design problem. This paper aims to present a definition, indicator(s) and an evaluation method for robustness against short-term variations in supply. However, the method could be extended to short term variations in demand. Besides this, it aims to position the term robustness in relation to other terms like reliability. By doing this, the paper gives policy makers and transportation analyst a framework to discuss issues that are related to road network robustness and vulnerability. Furthermore, the evaluation method that is presented contributes to the problem of designing robust road networks against short time variations in supply because it has a relatively short computation time and it takes queue spillback effects and alternative routes into account. The short computation time is required because the network design problem is a complex problem which requires many network evaluations since there are many possible robustness measures that can be applied on many different locations.

In section 2 of this paper an explanation is given of different terms that relate to robustness. The section thereafter explains how the robustness of a network can be assessed. Finally, in the last section some conclusions and recommendations are given.

\section{AN EXPLANATION OF TERMS THAT RELATE TO ROBUSTNESS}

\subsection{The context of robustness}

Figure 2 shows the relation between network characteristics and robustness and between robustness and reliable travel times. 
$<$ Figure 2 about here>

The numbers in the figure refer to the order in which the figure should be read:

(1) Under regular circumstances (no disturbances), the network performance is determined by the regular demand and supply pattern.

(2) Disturbances, such as accidents, special weather conditions, roadwork, events, and seasonalities, lead to short term variations in demand and supply. These disturbances occur with a certain probability and have a primary effect on the capacity (capacity reduction) and/or the demand (increase or decrease in demand).

(3) The primary effect of the disturbances combined with the regular demand and supply pattern results in a new level of demand and supply.

(4) The effect on the network performance (5) of this new level of demand and supply depends on the robustness of the network, the measures taken by network managers (e.g. information provision) and the response of drivers (e.g. route choice) to that. For instance, in a robust network deviations from the regular demand and supply pattern will result in less variation in travel time compared to a network with a lower robustness level. Robustness can be subdivided in five elements (prevention, redundancy, compartmentalization, resilience and flexibility) as is explained in section 2.4. Furthermore, as is explained by Nicholson et al. (2001), if the user has a high level of information (i.e. information is provided well in advance, and route guidance is available once the trip has commenced), the range of available options is greater and the consequence of degradation is reduced.

(6) The variation in travel time that results from the disturbances determines the objective travel time reliability. The variations in travel time can be expressed by a probability density function of travel time. Stability is the degree to which the travel time changes as the intensity rises and/or the capacity falls. Ideally, the change will remain limited; after all, a sudden large increase in travel times should be avoided, if possible. The way in which reliability (or unreliability) is experienced (8) depends on the characteristics of the driver (e.g. level of risk averseness), the trip purpose, the information that is received by the driver about disturbances, and the alternatives that are available to the driver. If the traveller is informed about delays and if route alternatives are available, then the travel times are more reliable in the perception of the driver.

Besides these relations, there are some other relations: The redundancy/spare capacity (element of robustness) depends on the regular demand and supply pattern. Furthermore, the response of the drivers to disturbances and measures taken by network managers depend on the characteristics of the driver, the trip purpose, the received information, and the available alternatives.

Above, the relation between reliability of travel times and a robust road network is illustrated. Although reliability and robustness have a strong relation, they are not are not identical. It is clear that robustness is a property of the system. By contrast, the reliability of the travel time is something that the traveller experiences. Immers et al. (2004) expressed this as follows: reliability is a user oriented quality while robustness is a characteristic of the system itself. In addition to this distinction, there are three other distinctions:

- Where reliability is concerned, the emphasis lies on disturbances that occur frequently, whereas with robustness the emphasis lies on disturbances that occur unexpectedly and that have a large impact. In Figure 3, a travel time distribution is shown in which the foci 
of reliability and robustness are indicated. A strict distinction can not be made, because unexpected disturbances can have a large effect (focus robustness) as well as a small effect. The same is true for frequently occurring disturbances (focus reliability). Therefore, the tail of distribution also has a small weight in the determination of the reliability of travel times. However, because very high travel times do not often occur, they are relatively unimportant for the reliability of travel times (they might have a large effect on the perception of reliability). On the other hand, road networks can be made robust against all kind of disturbances (disturbance with small and large effects). Because robustness focuses on unexpected disturbances, the tail of the distribution function becomes more important (unexpected disturbances have a higher effect than expected disturbances, in general).

$<$ Figure 3 about here>

- Reliability is geared towards an average spread in the travel time and, therefore, must be determined over a longer period (ranging from several days to a year). With robustness, the emphasis lies on the period in which the effect of a specific disturbance is noticeable. It focuses on the impact of single disturbances and not so much on the probabilities that these disturbances occur.

- In the case of reliability, the emphasis lies on the probability that a specific disturbance occurs, and with robustness the emphasis lies on the effect, as was also noted by others. Husdal (2004) noted that probability or predictability is a major concern in network reliability studies. The impacts or consequences of disturbances are the focus of vulnerability studies. D'Este and Taylor (2003) note that vulnerability and reliability are two related concepts, but emphasize that network vulnerability relates to network weaknesses and the economic and social consequences of network failure, not so much to the probability of failure. This distinction is, of course, related to the two previous points taken together. This does not mean that the probability of a disturbance is unimportant. For the functioning of the whole system, this probability is of great importance. If the probability that disturbances occur can be reduced, this will have a great effect on the average travel time and the reliability of the travel time. In this case, the robustness of the system is of less importance.

\subsection{Disturbances}

In the previous section it was explained that disturbances are the primary cause of unreliability and that a road network can be made robust against those disturbance which should result in more reliable travel times. This section elaborates on the disturbances that can occur and classifies them.

In traffic and transport many disturbance can occur which result in travel times that deviate from the travel times under regular conditions like natural disasters (e.g. earthquakes, hurricanes, floods, landslides), extreme weather, incidents, road works, social events (e.g. football matches, big fairs), malicious attacks and signal failures. There are many ways to classify these disturbances. In the literature about reliability quite often a distinction is made between recurrent (such as weekday peak hour congestion) and non-recurrent (such as floods and other events of nature) disturbances. The essence of the degree of recurrence is that it provides information about the predictability of the event. In Wilmink et al. (2003) a distinction is made between predictable and non-predictable conditions and between regular and non-regular conditions. Examples of regular and predictable situations are morning and 
evening peak hour congestion. Small incidents can be classified as regular non-predictable disturbances. Examples of non-regular predictable situations are holiday traffic, big events, and extreme weather conditions. Finally, in the class of non-regular non-predictable disturbances, we can mention calamities, big incidents, etc. Some of these disturbances influence the supply and others influence the demand.

Husdal (2004) classifies disturbances by their nature. He describes structure-related vulnerability, nature-related vulnerability, and traffic-related vulnerability. Structure-related or structure-generated vulnerability pertains to the way the road is built and attributes of the road network itself, not only in terms of topology, and connectivity, but also in terms of the physical, body of the road, geometry, width, curvature, gradient, tunnels, bridges, weight restrictions for certain vehicle types, etc. Nature-related or nature-generated vulnerability pertains to attributes of the natural environment, the topography and the terrain that the road traverses, and to nature-given incidents, such as flash floods, avalanches, rock fall, snow and ice, fog, earthquakes, tsunamis, and consequences of climate change, to mention but a few. Traffic-related or traffic-generated vulnerability pertains to attributes describing the generic flow of traffic and attributes resulting in flow decrements, such as daily rush hour and weekend highs, as well as maintenance operations, snow clearing, accident clear-up, and ongoing construction works. Besides these unintentional disturbances there are intentional disturbances, such as terrorist attacks.

Furthermore, a distinction can be made about the impact of the disturbances. Most disturbances have a temporary impact/effect, which can vary from small to large. However, there are also disturbances that have a permanent impact. Another distinction is between within day and between day variations. Finally, the location of the disturbances can vary. Some disturbances have a network-wide effect and others have a local effect.

The above mentioned distinctions match with risk theory: risk = probability $\mathrm{x}$ effect. Regular disturbances have a higher probability than non-regular disturbances. Furthermore, in general, non-regular disturbances have a higher effect than regular disturbances, and non-predictable disturbances have a higher effect than predictable disturbances, because preventive measures can be taken for predictable disturbances. In network design, both the probability and the effect are important. Of course, the disturbances that should get the biggest attention are those with both a high probability and a high effect. However, fortunately there are not so many of those disturbances.

If robustness issues are discussed, it is advisable to clearly indicate against which disturbances a network is to be made robust. As already explained, the robustness of a road network focuses more on effects than on probabilities. Nevertheless, for network design, probabilities are an important factor for investment decisions. Therefore, in this paper we focus on the class of non-predictable regularly occurring (relatively high probability) disturbances: incidents. We also consider some incidents, such as road closures, which belong in the class of non-regular non-predictable disturbances.

\subsection{Definition}

Above the relation between robustness and reliability is shown and an elaboration on the type of disturbances that can occur is presented. In this section we give a definition of robustness and reliability which build upon the previous sections. 
The most accepted definition of the network reliability is given by (Wakabayashi and Iida, 1992): Reliability is the probability of a road network performing its proposed service level adequately for the period of time intended under the operating conditions encountered.

In comparison with research into reliability, research into robustness and vulnerability is less extensive. The terms robustness and vulnerability have a strong relation, but they are actually each other's opposites. Vulnerability describes the weakness of a network and robustness describes the strength of a network. Berdica (2002) has done leading research into road vulnerability. She defines vulnerability in the following way: "Vulnerability in the road transportation system is a susceptibility to incidents that can result in considerable reduction in road network serviceability." In this definition the serviceability of a link/route/road network describes the possibility to use that link/route/road network during a given time period. Others also describe the vulnerability of road networks. For example, Taylor and D'Este (2003) relate vulnerability to the degree of accessibility of a given node in the network, where accessibility is expressed as the travel cost needed to access the particular node, comparing optimal and alternative routes or detours. D'Este and Taylor (2003) define vulnerability to be the likelihood of severe adverse consequences if a small number of links (or possibly a single link) is degraded. They distinguish between connectivity vulnerability and access vulnerability. Connectivity vulnerability considers a pair of nodes and the generalised cost of travel between them. If the loss or substantial degradation of one or more network links leads to a substantial increase in the cost, then the connection between those nodes is vulnerable. Access vulnerability considers a single node and the overall quality of access from that node to all other parts of the network. A node is vulnerable if the loss of substantial degradation of a small number of links results in a significant reduction in the accessibility of that node, as measured by a standard index of accessibility. It should be noted that the second definition of vulnerability ignores probability; this vulnerability is really a measure of the consequence of degradation (Nicholson et al., 2001).

Based on these definitions we came to the following general definition of robustness that considers the performance of a complete network and allows considering all kinds of disturbances on links, nodes, and routes that lead to a partial degradation of those elements or a complete loss of function of those elements: Robustness is the extent to which, under prespecified circumstances, a network is able to maintain the function for which it was originally designed. Vulnerability is the opposite of robustness. A network that is vulnerable is not robust, and vice versa.

Three elements of the above definition require more explanation:

- "Function": The most general function of a road network is to enable trips from origins to destinations. Achieving an adequate road network design requires knowing for which kind of trip a network link or network node has a function. Trips can be categorised by their length, whether they are for passenger or freight transport, and by their purpose. The level at which a network has to function is usually specified by a government organisation or defined by design standards.

- "Pre-specified circumstances": In the previous section it is explained that a network can be made robust against all kind of circumstances. However, in practice, choices have to be made by policy makers about the disturbances on which they want to focus. In this paper we focus on short term variations in supply caused by incidents.

- "The extent to which": The definition includes the words "the extent to which". This implies that, in cases of disturbances, the network does not have to function just as well as it would without disturbances. From an economic perspective it is not advisable to make a network $100 \%$ robust against 
all disturbances. The costs of creating such a network would exceed the benefits. However, this does raise the question of the extent to which the network should maintain its function. This is a question that needs to be addressed by policy makers and network managers as well. A balance has to be found between investments in robustness measures and reliability benefits for travellers.

\subsection{Elements of a robust road network}

To get a better understanding of robustness we can ask ourselves the question: "What makes a network robust?" or the other way around: "What makes a network vulnerable?" Answering these questions not only clarifies the term robustness, but also gives direction for specifying indicators for robustness and for the measures that need to be taken to make the network more robust. In order to answer these questions, we made an analysis of all the incidents that occurred in the region South Holland in the Netherlands during the period January 1st - April 15th 2007. In total, 3484 incidents were considered. Of these 3484 incidents, 1046 were accidents and the other 2438 were car or truck breakdowns. The incident information (location, start time, end time, date, and incident type) comes from the "Program monitoring incidents from DVS". We combined a database with incident information with traffic counts on the motorways. From the flow and speed data, the vehicle loss hours can be estimated by using the "piece-wise linear speed-based algorithm (PLSB)" (Van Lint and Van der Zijpp, 2003). Since congestion can also occur when there are no incidents, we computed the vehicle loss hours that occur during four reference days. The reference days were four days in the same week (for weekdays). For Saturdays and Sundays, four weekend days were used as a reference. The vehicle loss hours caused by an incident are computed by subtracting the vehicle loss hours of the reference days from the vehicle loss hours that occurred during the period in which the effects of the incident were noticeable. The vehicle loss hours of the incident and the reference days are computed on the road on which the incident occurred. Of course, the traffic jam can also spill back to other roads. However, these spillback effects are not considered explicitly. Furthermore, the congestion can spill back to local roads. The congestion on these roads is not measured either, since data for these roads was not available. Finally, we cannot be sure that incidents did not occur on the reference days. These are three reasons why the vehicle loss hours of incidents have been underestimated. Nevertheless, the results give a good indication of vulnerable road sections. Jonkers et al. (2009) explain the method that was used in more detail for truck incidents. A similar approach was used for all incident types

In Figure 4, the number of incidents that occurred on a certain motorway road segment is displayed. Figure 5 shows the average effect expressed in vehicle hours lost on these road segments. Finally, Figure 6 shows the total number of vehicle loss hours per kilometer as a result of incidents that occurred on each road segment. Although, quite a lot of incidents were included in the analysis, when interpreting these incidents it has to be taken into account that the incidents vary in severity. Due to the inherent randomness of incidents, some incident locations might by chance be indicated more or less vulnerable than they would be had we analyzed more incidents over a longer period of time.

$<$ Figure 4, 5, 6 about here>

In the mapping of the vehicle loss hours, we had to deal with the following limitations:

- An incident is characterized by a road number and a hectometre number. However, multiple road segments in the GIS-layer can have the same road number and hectometre 
number. This happens, for instance, on motorways with parallel road structures or at junctions. Therefore, the same incident can be plotted on multiple road segments.

- One road segment can be longer than one hectometre. Therefore, incidents that occurred on different locations may have been plotted assigned to the same road segment.

We combined the figures above with our knowledge about the network (especially the secondary network) and the locations on which regular congestion occurs. This resulted in the following, not necessarily new, hypothesis:

1. Incidents that occur on roads with a high intensity-capacity ratio (I/C-ratio) or a high intensity-spare capacity ratio (I/(C-I)-ratio), and thus with little spare capacity, have a higher effect (expressed in vehicle loss hours) than incidents that occur on roads with low I/C-ratios.

2. Incidents that occur on roads with high flow have a higher effect (expressed in vehicle loss hours) than incidents that occur on roads with lower flow, if the I/C-ratio is equal.

3. Incidents that occur at locations where good alternative routes are available have a lower effect (expressed in vehicle loss hours) than incidents that occur at locations where good route alternatives are not available.

4. Incidents that occur near intersections are likely to have a larger effect (expressed in vehicle loss hours) than other incidents.

5. Merge locations and bridges have a higher chance of incidents than other locations.

Furthermore, an analysis of the relation between the duration of the incidents and the effects of the incidents (expressed in vehicle loss hours) shows that a strong linear relation between duration and effect does not exist $\left(\mathrm{R}^{2}=0.11\right)$. This can be explained by the fact that the impact of an incident depends not only on the duration, but also on other factors, such as spare capacity on the link where the incident occurs and on alternative routes, spillback effects, etc. With respect to the duration, Knoop (2009) analytically derived a formula for the total delay when all factors other than the duration are kept constant. He showed that if spillback effects are not considered, the delay is proportional to the square of the blocking duration. In case spillback occurs, the delay grows faster than proportional to the duration squared. This results in the sixth hypothesis:

6. Incidents with a long duration are more likely to have large effects than incidents with a short duration.

\section{Elements of a robust network}

The data about incidents is not detailed enough to test these hypotheses statistically. Although, the analysis of incidents does not prove that the hypotheses are true, we do generalize the hypotheses to the following five elements that are likely to make a network more robust:

1. Prevention: the road system will continue to function well if disturbances are prevented. However, the concept of prevention is not used here in relation to the robustness of the network in the sense of the prevention of disturbances, but the prevention of congestion due to disturbances. For example, if the road surface is heated, then snow and freezing rain are less likely to cause problems on the road. Furthermore, better driver training could result in a better response of drivers in case of disturbances, and therewith to less vehicle loss hours as a result of the disturbance.

2. Redundancy: the robustness of a system can be increased by introducing a certain spare capacity into the system (e.g. Goodwin, 1992). This spare capacity is often referred to by the term 'redundancy'. When disturbances occur, this spare capacity can be made available so that the system continues to function better. There are actually 
two types of redundancy: active and passive redundancy. Active redundancy, like alternative routes, is redundancy in the network that can also be used in regular situations. If redundancy in the network is to be created, also measures (e.g. road pricing, or speed adjustments) are necessary that preserve the spare capacity under regular conditions. Passive redundancy refers to back-up options that are only used in case of disturbances. An example of this are ferries that can be used in case of bridge failures. (This element relates to hypotheses $1-3$.).

3. Compartmentalization: this is the degree to which traffic congestion remains restricted to the relevant link or a small section of the network. If there are less interdependencies in the network, congestion at a centrally located link or node will not cause a series of cascading failures disrupting traffic on large parts of the networks. (This element relates to hypothesis 4.)

4. Resilience: Resilience is the capability of the transport system to recover, preferably within a short time period, from a temporary overload. (This element relates to hypothesis 6.)

5. Flexibility: the robustness of the system can partly be measured by the degree to which the system is able to fulfil more and different functions than the functions for which the system was originally designed. In other words, flexibility is a property that enables the system to expand in line with new requirements that are demanded of the system.

Four of these elements have already been mentioned by Immers et al. (2004). Furthermore, in other disciplines these elements can be recognized as well, which strengthens the conclusion that a road network is more robust if it includes the above mentioned elements. In railway networks, many preventive measures (such as fences and heating parts of the rails) are taken in order to prevent disturbances (like snow) from having large effects. In the nervous system, the nerves are very well protected, which is an example of a preventive measure. In the Internet, there is a lot of redundancy in the cable network (many alternative routes are available). In airplanes, there is redundancy in the number of engines. Fire doors in buildings are a good example of compartmentalization. The cardiovascular system is an example of a very resilient network. The diameter of the blood vessels can instantaneously be adjusted is such a way that the brain and heart will keep receiving enough oxygen to function properly. And blood vessels are an example of flexibility as well, since the capacity of the blood vessels can be varied.

\section{A FRAMEWORK FOR ROBUSTNESS ANALYSIS}

\subsection{Indicators}

In order to measure robustness we need to define one or more indicators for robustness. Of course the quality of the indicator(s) is very important. Below a set of questions/criteria are listed that can be used to score the indicators:

1. Does the indicator describe the concept of robustness in a complete (all elements of robustness) and logical way? In other words: is there face validity? If the indicator increases, is the network more vulnerable (=less robust), and vice versa?

2. Are there data available for monitoring the indicator?

3. Can the indicator be estimated inside a computer model?

4. Can the indicator be estimated inside a computer model within an acceptable computation time? 
5. Can the indicator be explained to policy makers and other people who are not robustness experts?

6. Can the indicator be calculated on the network, route and link level?

7. Can the indicator be evaluated in cost-benefit analysis?

The more of these questions that can be answered positively for a specific indicator, the better the indicator is suited for robustness analysis and robustness optimization.

Up to now, no generally accepted indicator for robustness exists. The list below contains indicators that can be used to determine the robustness of a network. This list of indicators is mainly based on (Murray-Tuite and Mahmassani, 2004), (Tampère et al., 2007), (Li, 2008), and (Jamakovic et al., 2008). Of course, others use similar indicators. Furthermore, there might be other robustness indicators that are not included in this list. We classified the indicators in the following way. The static (i.e. independent of the traffic flow) indicators refer directly to the properties of a network, and therefore to the robustness of the network. The dynamic (i.e. dependent on the traffic flow) indicators refer directly to the robustness of a network. Finally, the indirect indicators refer to the travel time and to the stability of the travel time. Between parentheses, the elements of robustness to which the indicators are related are mentioned.

\section{Static indicators}

1. The availability and quality of alternative routes (redundancy). If allowance is made for the traffic intensity on the alternative routes, this indicator becomes a dynamic one. An example of such an indicator is the vulnerability index (Murray-Tuite and Mahmassani, 2004). An aggregation of the vulnerability index across all origins and destinations results in the 'disruption index'. The disruption index accounts for the availability of alternate paths, travel times, marginal costs, and link capacity (Murray-Tuite and Mahmassani, 2004).

Furthermore, we propose an additional indicator for alternative routes. This indicator is presented in equation 3.1 .

$$
\text { Altroutes }_{\mathrm{a}}=\frac{c a p_{a}}{\sum_{a a \in A_{a}}\left(\operatorname{cap}_{a a} * \varsigma 1^{\text {dista,aa }_{a}}\right)}=\frac{\operatorname{cap}_{a}}{\sum_{a a \in A_{a}}\left(\operatorname{cap}_{a a} * e^{\varsigma 2^{*} d i t_{a, a a}}\right)} \quad(\varsigma 2=\ln (\varsigma 1))
$$

In this formula, $a$ is the link where the disturbance occurs, $a a$ is a link from the collection $A_{a}$ of links that form an alternative for link $a$, cap is the link capacity, $\varsigma 1$ and $\varsigma 2$ are parameters that represent the importance of the distance from alternative routes, and $d i s t_{a, a a}$ is the shortest distance over the network between link $a$ and link $a a$. The set $A_{a}$ is determined by taking a line perpendicular to the mid-point of link $a$. In Figure 7 an example of such a line is shown. The links that cross the gray line with the black dots are considered to be an alternative for the black line with the white dots (also indicated with an arrow) if they meet the following requirements:

- The absolute angle between the original link and the alternative link must be smaller than 60 degrees. Of course, this parameter can be varied. The choice for 60 degrees was made in such a way that routes that do not run more or less parallel are not considered as alternatives.

- The direction of the original link and the alternative link must the same (e.g. if one link runs in the north-south direction, the alternative link must also run from north to south. Links in the opposite direction south-north, are not considered).

By multiplying the capacity of the alternative link by the parameter $\varsigma l$ with the distance between the two links as the exponent, nearby links are considered more important than more 
distant links. $\varsigma 1$ must have a value between 0 and 1 . We chose to set $\varsigma 1$ to 0.8 , which implies that links up to about 10 kilometres are considered to be valid alternatives $(\varsigma 2=\ln (\varsigma 1))$. This parameter could also be varied. In areas where people are used to making longer trips, this parameter could be set a bit higher. In an alternative formulation, $\varsigma 1$ is replaced by $\exp (\varsigma 2)$. Another choice that could be made is to make $\varsigma l$ trip distance specific. For long distance trips, route alternatives that are far away from each might still be good route alternatives, whereas for short distance trips, far away route alternatives are not good alternatives, since they would result in large detours. In the literature, we could not find any statistical evidence about which detours are acceptable to drivers in incident situations. We chose a maximum of 10 kilometres, because this seemed to be a reasonable distance given the fact that almost $50 \%$ of the traffic in the Randstad on the motorways has a trip distance shorter than 20 kilometres (4Cast, 2005). We do not make a distinction between distance classes, because for both shortand long- distance travellers, the far away options are only alternatives when they have not past the point where the original and the alternative route split at the time when they are informed about the incident. If an alternative link has a higher capacity it is considered to be a more useful alternative. The flows on the alternative route are not considered. Including the capacity of link $a$ ensures that links with a higher capacity are given a higher score. The higher the score, the more vulnerable the link is. Links with a score higher than 1 are considered vulnerable. The indicator defined above gives an impression of the vulnerable links in the network solely based on the network structure and can be computed within a short computation time.

\section{$<$ Figure 7 about here>}

This indicator could easily be extended to a time dependent indicator that is based on flows and spare capacity in time and space. In equation 3.2, an extension of this indicator is shown. Here, $v_{a}$ is the flow on link $a$ and $r c_{a a}$ is the spare capacity on link $a a$. This indicator can be calculated for different time intervals or for a longer period. Finally, care should be taken when the spare capacity is computed by subtracting the intensities from the capacities, since low intensities can refer to congested and non-congested states. For instance, if a road is completely congested, the intensity is $0 \mathrm{pcu} /$ hour, which indicates that there is a lot of spare capacity in the network, whereas in practice there is not even space for one extra car.

Therefore, a correction should be applied. This can, for instance, be done by setting the spare capacity to $0 \mathrm{pcu} /$ hour if the speed ratio drops below a certain threshold.

$$
\text { Altroutes } 2_{\mathrm{a}}=\frac{v_{a}}{\sum_{a a \in A_{a}}\left(r c_{a a} * e^{\varsigma 2^{*} d i t_{a, a a}}\right)}
$$

2. Graph theoretical measures, such as:

- The degree (distribution): a node's degree describes the number of neighbours a node has. The nodes' degree distribution is the probability that a randomly selected node has a given degree (compartmentalization, flexibility).

- The distance (distribution): The distance distribution $P($ dist $)$ is the probability that the length of the shortest path between a random pair of nodes is dist.

- The clustering coefficient: The clustering coefficient of a node is the proportion of links between nodes within the neighbourhood of a node, divided by the maximum number of links that could possibly exist between those neighbours (redundancy). 
- The connectivity in a network: the link connectivity is the minimum number of links whose removal would disconnect a graph. The node connectivity is defined analogously (nodes together with adjacent links are removed) (redundancy).

- The centrality/the betweenness: betweenness is a centrality measure of a node (link) within a graph: nodes (links) that occur on many shortest paths between other node pairs have higher node (link) betweenness than those that do not (redundancy).

- The coreness: The $k$-core of a graph is a subgraph that is obtained from the original graph by the recursive removal of all nodes of degree less than or equal to $k$. The node coreness of a given node is the maximum $k$ such that this node is still present in the $k$-core but removed from the $(k+1)$-core.

3. The distance between on ramps and off ramps (compartmentalization, flexibility).

\section{Direct dynamic indicators}

4. Spare capacity. This is the capacity that is not used in normal circumstances (redundancy).

5. The total length of the roads on which the consequences of a disturbance are noticeable (compartmentalization, redundancy).

6. The average time before an incident is resolved (resilience).

7. The total number of vehicles on the roads on which the consequences of a disturbance are noticeable (compartmentalization, redundancy).

8. The total distance covered by all vehicles over a whole period in the situation with an incident compared with that situation without an incident (redundancy).

9. The total number of arrivals in a specific period in the situation with an incident compared with that situation without an incident (all elements).

10. The number of vehicles on the network in a period in the situation with an incident compared with that situation without an incident (all elements).

\section{Indirect dynamic indicators}

11. Total travel time of all vehicles per time interval in the situation with an incident compared with that situation without an incident (travel time, stability).

12. The extra travel time caused by an incident (travel time).

13. The average speed per time interval in the situation with an incident compared with that situation without an incident (travel time, resilience, and stability).

Most of these indicators can be determined at the road section level, route level, and network level. Indicators at network level tell us something about the functioning of the whole network. Indicators at network level are of particular importance to the network administrator. With indicators that are calculated at network level, it must be taken into account that a small change in, for example, the total travel time of travellers that make use of a network can still have a big impact for individual travellers. A local incident will, for example, reduce the total network performance by only a small percentage (for example $0.5 \%$ ). This is due to the fact that the incident does not have any effect on a large part of the network, and because the incident causes congestion for only part of the time. However, the individual traveller that finds himself in the middle of the congestion due to that incident can easily suffer an increase in travel time of perhaps $50 \%$. By calculating the indicators at route level, it is possible to determine which road users suffer from an incident (or other disturbances). By selecting all routes that run past that incident location, the losses in travel time for the travellers affected by the incident can be made transparent. This is done by zooming in on a part of the travellers and a part of the travel time. Therefore, the effect of a disturbance is magnified. By focusing 
on the road section level, we can determine road sections on which the effect of incidents is noticeable. This means that the effect of a disturbance is even more magnified.

In addition to the distinction made between road sections, routes, and networks, we can also make a distinction between periods. It is possible to determine some indicators for whole days, parts of days, or time intervals (hours or even minutes). This last-mentioned aspect also makes it possible, for example, to determine how quickly the network 'collapses' after disturbance, and how quickly it recovers again. Finally, for indicators where traffic flow plays a role, a distinction can be made between passenger transport and goods transport and between short distance travellers and long distance travellers. Long distance travellers have, for instance, more route alternatives available than short distance travellers, if they are informed about disturbances in time and if they are not too far on their route to be able to switch to the other route. Therefore, it might be needed to use a different value for the parameter $\varsigma 2$ in equation 3.2 for long distance traffic and for short distance traffic.

As indicated above, a single universal indicator for robustness does not exist. The various indicators defined above all provide a picture of some aspect of robustness. Indicators 1 to 10 refer directly to robustness, and are therefore of importance in the design phase. Indicators 11 to 13 are related to travel time, and are therefore easier to use in cost-benefit analyses, because evaluations exist for travel time. Of course it is ultimately of importance how measures that have been formulated in the design phase to improve robustness affect travel time, reliability, and the loss in travel time as a result of disturbances.

For the analysis presented in the remainder of the paper, we chose to use the indicator vehicle loss hours caused by incidents (indicator 12) because:

- This indicator describes the different elements of robustness. Depending on the way it is computed, the indicator can consider spillback effects (compartmentalization), route alternatives (redundancy), and resilience. If flexibility is included in the network, these flexible infrastructures can be considered (depending on the type of model that is chosen). The element 'prevention' relates to the capacity reduction that occurs as a result of incidents. This can be included in the input of the model that is used (criterion 1).

- Monitoring this indicator is difficult. It requires a link between incident registration databases and traffic counts, as is done in the previous section. For a complete picture, traffic counts on the secondary network are needed as well. The most difficult part is the data about the reference day, because reference days do not exist (criterion 2).

- Modelling this indicator is also difficult. Ideally a dynamic model is needed to model spillback effects of incidents properly (Knoop, 2007 and 2009). There are many different disturbances that can occur on many different locations of which ideally the effects should all be modelled by using this dynamic model. Finally, there is a lot of uncertainty about the choice behaviour (route choice, departure time choice, and mode choice) of travellers in case of disturbances. The amount of information given to the traveller can vary, as well as their responses to it. The next section describes the way in which this indicator can be modelled in the best possible way. In the next section it is explained that we use the alternative route indicator (equation 3.2) in the computation of the vehicle loss hours caused by incidents (criterion 3 ).

- The indicator is relatively easy to explain, because policy and decision makers are used to thinking in terms of travel time losses. However, it must be clearly stated that not only the travel time delays of people that are in the queue caused by the incident should be considered. The travel time losses as a result of taking detours (of the people that take the 
detour and of the people that were on the route of the detour) should be considered as well (criterion 4).

- In order to compute the indicator on the network level, the chance of disturbances is needed. Multiplying the chance of all disturbances that are relevant for robustness by the effects of those disturbances results in the total expected travel time loss on the network in a certain period. If different networks (for instance, without and with a robustness measure) are compared, it must be taken into account that measures could for instance result in extra vehicle kilometres driven as a result of distribution effects that could result in lower robustness scores. The construction of an alternative route is, for instance, expected to improve the robustness of the network. However, if this route is fully used in the regular situation, it does not offer spare capacity in case of disturbances on other routes. Because the number of vehicle kilometres driven on the network increased, more people are delayed in case of disturbances, and the chance of a disturbance is larger (criterion 5).

- The indicator can be evaluated by using values of time. However, a value of time in case of unexpected disturbances is not yet available. There is a lot of research into values of reliability. Therefore, it is likely that in the future there will be value of time for travel time losses caused by unexpected disturbances (criterion 6).

As can be seen from the list above, there are still some problems in monitoring and modelling this indicator. Nevertheless, this indicator was chosen because the indicator scores well on the other criteria. Since, there is not one indicator that scores well on all the criteria, a choice was made to prefer an indicator that well describes the concept of robustness, is explainable, can be used to obtain a network wide indicator and can be used in cost-benefit analysis, but has some technical (data and model wise) challenges, over indicators that can easily be measured and modelled, but do not have explanatory power.

Choosing for travel time losses as a result of disturbances as our primary indicator of robustness does not imply that the costs of extra vehicle kilometres driven in case of disturbances should not be considered. However, the costs of extra vehicle kilometres driven are expected to be much lower than the costs of travel time losses, because, depending on the severity of the incident, only a small percentage of the travellers take a detour (e.g. Knoop (2009)) and because time-related costs are usually higher than distance-related costs (when road pricing is introduced this might change). The indicator chosen can be extended to generalized cost (a composite distance and time related cost).

\subsection{Evaluation method for robustness}

Given the elements of robustness described above, ideally a method that is used should meet the following requirements:

- Spillback effects should be modelled. Spillback effects are the cause of the fact that the effects of local disturbances spread all over the network. In a robust network, these effects are minimized (for instance by creating compartmentalization). If robustness is to be assessed, these effects should be captured.

- Alternative routes should be included in the route choice. A network is more robust if alternative routes are available since they offer spare capacity that can be used in case of disturbances. Furthermore, they create a balanced network.

- Time dynamics should be included. Since the speed at which network performance drops during disturbances and the speed at which the network recovers after disturbances are important for the robustness, time dynamics should be included. 
- The method should be fast: since measuring robustness requires a lot of simulations of different disturbances on different locations, a method with a short computation is preferable.

- The method should be able to deal with all kind of disturbances in such a way that the complete travel time distribution is modelled.

- The method should be able to deal with intersection delays, because in a robust road network, regional (and local roads) are important elements.

The dilemma in the model choice is that a choice has to be made between accuracy (ideally using a dynamic traffic assignment model with detailed congestion modelling, including spillback effects, with multiple types of route choice behaviour during incidents, and with an accurate intersection modelling) and computation time. In general, the most accurate models have the longest computation time. Using rules of thumb takes hardly any computation time, but is not accurate. For some applications, a rule of thumb can be good enough to get a quick impression of the robustness of a network. However, to make a well balanced decision about robustness measures in network design, it would be better to look for a method/model that deals with the above requirements in the best possible way.

To the best of our knowledge, there is not yet a model that covers all these six features completely. We chose to use the macroscopic dynamic traffic assignment model 'Indy' (Bliemer, 2007), because this model has an accurate network loading model that models spillback effects according to the simplified kinematic wave theory of Newell (Yperman, 2007). Furthermore, the model can compute an equilibrium route choice and can deal with fixed route choice. En-route route choice is not possible. However, this is not a problem, since Indy is used only for a basic run in a situation without disturbances, for which an equilibrium assignment is most appropriate. Indy is a dynamic model, which makes the modelling of time dynamics possible. In order to model the effects of incidents, a marginal incident computation model (MIC) (Corthout et al., 2009) has been attached to Indy. The MIC-module is able to get an estimate of the impact of incidents very quickly. The MIC-module is currently capable of dealing only with fixed route choice during incidents, which can be considered as a disadvantage. However, to partly overcome this shortcoming, we used an approximation method for the use of alternative routes. Finally, the MIC-module can only simulate the effects of local capacity reductions. Therefore, variations in demand and network-wide capacity variations (for instance as a result of rain) cannot be modelled with the MIC-module. If the impact of these disturbances is to be simulated, a complete run with Indy has to be done, which results in extra computation time. Finally, Indy does not have an explicit modelling of intersections and traffic signals. Therefore, it underestimates the delays at intersections. In (Snelder, 2009), it is shown, that to a certain extent, delays at intersections can be approximated by using outflow constraints that reflect the capacity constraints at intersections.

Given these arguments we came to the following two step evaluation method: In the first step an equilibrium assignment is done with Indy. Among others, this results in the cumulative inflows and outflows per link to all other next links. This is used as an input for the MICmodule (step 2). Furthermore, the MIC-module needs to know on which link what kind of incident occurs. We choose to model four types of incidents on all links. As is shown in (Knoop et al., 2010), it is very difficult, if not impossible, to make a pre-selection of the most vulnerable links. Therefore, the choice was made to use this full computation method. We model the following four incident types: 
- Car break down with a duration of 15 minutes a capacity reduction of 5\% and a chance of occurrence of 0.34 per 100.000 vehicle kilometers.

- Small incident which blocks one lane with a duration of 45 minutes a capacity reduction of $20 \%$ and a chance of occurrence of 0.06 per 100.000 vehicle kilometers.

- Big incident which block more than one lane with a duration of 45 minutes a capacity reduction of $70 \%$ and a chance of occurrence of 0.015 per 100.000 vehicle kilometers.

- Rubbernecking, with a duration of 45 minutes a capacity reduction of $15 \%$ and a chance of occurrence of 0.07 per 100.000 vehicle kilometers.

These incident types with the matching incident probabilities, durations and capacity reductions are based on the statistical analysis that is carried in SMARA (Meeuwissen et al., 2004). In SMARA, however, a distinction is made for different road types, and roads with one, two, three or more lanes. It is for instance logical that the capacity reduction of the third incident type depends on the number of lanes. Since the input of the MIC-module is link specific, this distinction can be made here as well without any problems. The numbers above are presented to give an impression how the model works. In practice all the input files should be adjusted to the specific situation anyway.

The probabilities of the incidents are not needed to compute the effects. Strictly speaking, they are not input to the MIC-module. However, they are needed to compute the expected vehicle loss hours in a certain period. Finally, also the start time and end time of the incidents are needed as an input. The number of vehicle loss hours of an incident is, for instance, different in the peak period than in the off-peak period. In order to keep the computation time within acceptable limits, the number of periods for which an incident is computed should be kept as limited as possible. Choosing some representative incident types (chance, duration, reduction, and start time) is therefore always advisable. It is up to the model users to do this in the most appropriate way.

The output of the method is the number of vehicle loss hours per incident per link. The output also indicates which links are affected by each incident. Furthermore, the vehicle loss hours are also given per route. Multiplying the vehicle loss hours by the incident probabilities gives the expected vehicle loss hours.

The fact that both Indy and the MIC model are not suitable for assessing the usefulness of alternative routes can be considered as a serious shortcoming, because alternative routes are an important part of robustness. Therefore, for both models we implemented a simple algorithm that gives an approximation of what will happen if alternative routes are used in incident situations. Both methods need the maximum percentage $(x \%)$ of drivers that choose an alternative route during incidents as an input.

For Indy we implemented a path shift algorithm. At first an equilibrium assignment is done for the case without disturbances. From this we know the available paths, path departure flows and path travel times. Drivers only choose this alternative path if it doesn't have a travel time larger than their original travel time $+y \%$. Furthermore, they only choose an alternative path if it makes sense given the time of the incident and the time at which they pass the splitting point between their original path and the alternative path. The car drivers should be before the point at which their original path and the new path split or they should still be at their departure location when the incident occurs and the information about this reaches the driver (the time for spreading information is a parameter in the model). From all the drivers that can choose an alternative path $x \%$ switches to all suitable alternative paths. Based on this 
algorithm the path departure flows are changed and a new simulation with these path departure flows is done (only 1 iteration with Indy). A second version of the path shift algorithm starts with an equilibrium assignment and, thereafter, simulates the effects of an incident by assuming that everybody sticks to their original path (only 1 iteration with Indy). The resulting path travel times can be compared with the travel times of the case without disturbance. The people with a delay larger $z \%$ will choose an alternative path if they meet the other criteria above. This module requires extra calculations and an extra simulation with Indy (1 iteration) and is therefore slower than the first algorithm. However, it is more accurate since in this way also people that don't pass the incident location can change their route if they experience delays as a result of spillback effects of incidents. In the first algorithm only the people that actually pass the incident location can choose alternative routes. Another extension of the model could be a refinement of which alternative paths are actually chosen. In the current version all alternative path get assigned an equal amount of drivers from the blocked path. However, some alternative paths will always be preferred over others since they have shorter travel times.

The above algorithm illustrates how difficult it is to correctly model the behavior of drivers. A lot of assumptions have to be made by the modeler. Therefore, it is advisable to use the model with different parameter settings. This could be seen as different scenarios with respect to the information that is given to drivers and the way they respond to that.

For the MIC-module, we use an even more simplified algorithm. In this algorithm, the maximum available spare capacity on the alternative routes ${ }^{1}$ and the percentage of drivers that choose an alternative route $(x \%)$ is added to the capacity of the incident location (The jam density and the capacity reduction are adjusted as well.) If, for instance, $15 \%$ of the traffic that passes an incident location chooses an alternative route, the intensity on the original link is $15 \%$ lower. The effects of this can be approximated by not reducing the intensity by $15 \%$, but by increasing the capacity by the same number of vehicles as the $15 \%$ reduction. In this way, the number of travellers that experience a delay as a consequence of the incident is the same, and the spillback effects are the same as long as the congestion does not spill back over a node. If the congestion does spill back over a node, the capacity reduction is passed on to the following links in a different way. This is shown in the example below

\section{Example:}

A node has one incoming link and two outgoing links - one that goes to the incident (route A) and one that goes to the alternative route (route B). In the regular situation, the flow is 3450 in the direction of the incident route and 550 in the direction of the alternative. In case of an incident, a capacity restriction of 2000 pcu/hour due to an incident could result in a new distribution of flows: 3000 on the first route and 1000 on the second. The outflow in the direction of the incident is then restricted to $2000 \mathrm{pcu} /$ hour due to the incident, and the outflow to the other direction is restricted by the same ratio to $667 \mathrm{pcu} /$ hour in the node model of Indy. In the approximation that we use, the same incident leads to a capacity restriction of 2450 vehicles per hour $(2000+450$ vehicles that choose an alternative route). The incident restricts the flow in one direction to $2450 \mathrm{pcu} /$ hour and in the other to $391 \mathrm{pcu} / \mathrm{hour}(=$ $2450 / 3000 * 550)$. The flow further upstream in this approximation method is therefore restricted to $2841(2450+391) \mathrm{pcu} /$ hour instead of $2667 \mathrm{pcu} / \mathrm{hour}$. This example illustrates

\footnotetext{
${ }^{1}$ The available spare capacity on alternative routes is determined similar to the procedure used for calculating the altroutes index (section 3.1.): $r c_{\mathrm{a}}=\sum_{a a \in A A_{a}}\left(r c_{a} * e^{\varsigma 2 * d i s t_{a, a a}}\right)$
} 
that the spillback effects are not modelled $100 \%$ correctly in the approximation that we use. Furthermore, possible delays on the detours are not considered either. These are not only delays as a result of possible extra congestion on the alternative routes for the original and new users, but also extra travel time as a result of taking an alternative route that has (also without the extra congestion) a higher travel time than the original route, otherwise the alternative route would have been chosen in the first place. In the future, the MIC-module will be improved in such way that it can better deal with alternative route choice. For now, this approximation is used, since it accounts to a certain extent for additional benefits if alternative routes are available. Furthermore, we believe that the extra error that is introduced is not bigger than the error that we make anyway by not knowing how people behave during incidents.

It is important to know what the quality is of the modelled effects of incidents if the framework mentioned above is used. Therefore, Indy has been calibrated for the regular situation on the network of the area Rotterdam-The Hague in the Netherlands. Thereafter, five incidents with Indy were modelled by using different percentages of travellers that choose an alternative route (using the first path shift algorithm) and compared the effects with the available data about the incidents. An extensive description of this comparison and the results is given in (Muller, 2009). It was indicated that every incident is different. Forecasting the impacts is therefore extremely difficult. However, the results showed that the model reasonably approaches reality (root-mean-square percentage error between 0.2 and 0.3 ). The best fit was found when $15 \%$ of the road users changed their route as a result of an incident. The performance of the MIC-model has not been tested for the same incident situation, however for the Sioux Falls Network it was shown that the average deviation in vehicle hours lost is only $0.9 \%$ compared to incident simulations with fixed route choice in Indy (Corthout et al., 2009).

\section{DISCUSSION, CONCLUSION AND RECOMMENDATIONS}

If policy makers and transport analyst want to design a robust road network a lot of choices have to be made. For a start, there has to be common understanding with respect to the term robustness: What is it? Against which disturbances should the road network be made robust? How robust should the network be? Which indicator for robustness is to be used? How can robustness be evaluated? In a later phase the following questions have to be answered: Which measures can be taken to improve the robustness? What are the benefits of those measures and how do they relate to other benefits and costs? What should the robust network design look like?

In this paper we presented a framework for analyzing the robustness of a road network. The framework defines robustness, the elements of robustness and the relationship with related terms like disturbances and reliability. The framework is setup in such a way that it can be used in network design. The paper contributed to the existing literature by giving a overview from definition to evaluation method for robustness. Many choices that have to be made are mentioned and for some of them suggestions were presented.

We proposed to use the following definition for robustness: Robustness is the extent to which, under pre-specified circumstances, a network is able to maintain the function for which it was originally designed. Vulnerability is the opposite of robustness. A network that is vulnerable is not robust, and vice versa. It was explained that policy makers and/or network managers have to define themselves what the function of their network is for which group of users and to which extent this 
function has to be maintained in case of different kind of disturbances. In this paper we focused on short term variations in supply (incidents), but of course the network can also be made robust against other disturbances.

Furthermore, it was explained that robustness consists of five elements: prevention, redundancy, compartmentalization, resilience and flexibility. When the robustness of a road network is evaluated these elements have to be considered in the best possible way. We proposed to use the indicator 'travel time losses caused by incidents'. Finally, we proposed to evaluate the chosen indicator by using a macroscopic dynamic traffic assignment model in combination with a marginal incident computation model and an approximation method for taking into account alternative routes. In this way many incident scenario's can be evaluated by taking into account spillback effects and alternative routes within a short computation time. After the first equilibrium simulation run, hundreds of incidents can be evaluated within minutes.

The framework that is presented in this paper offers a common starting point for further discussion. However, it is clear that a lot of work still has to be done in order to be able to evaluate robustness with all its elements and in order to be able to make network designs that are robust against short term variations in demand and supply:

- Guidelines will have to be made in which the impact of different choices, for instance with respect to the disturbances that are considered and the level at which a network should maintain its function in case of disturbances, are made more comprehensible.

- Methods for analyzing the robustness from data (monitoring) should be improved in such a way the impact of different disturbances on the travel times can be monitored.

- The evaluation methods should be improved in such a way that the effects of disturbances other than incidents can be modelled as well within acceptable computation time.

- The route choice modelling in the marginal incident computation model should be improved and more information is needed about the actual route choice behavior of people in case of disturbances.

- Information about other choices (beside route choices) in case of disturbances (like staying at home, choosing another destination or changing the departure time) is needed as well. This information should be included in the evaluation method.

- Finally steps will have to be made in including robustness in cost-benefit analysis and network design.

\section{ACKNOWLEDGMENTS}

This research is supported by the Dutch research programs Next Generation Infrastructures, TRANSUMO-ATMA and TRANSUMO-NiVeS. The authors also thank the reviewers whose comments and text suggestions have been incorporated in the paper.

\section{REFERENCES}

4Cast (2005) Analyse HWN/OWN verkeer met het LMS, Rapport voor de Adviesdient Verkeer en Vervoer, Leiden, The Netherlands.

Berdica, K. (2002) An introduction to road vulnerability: what has been done, is done and should be done. Transport Policy, 9, 117-127 
Bliemer, M.J.C. (2007) Dynamic Queuing and Spillback in an Analytical Multiclass Dynamic Network Loading Model. Transportation Research Record: Journal of the Transportation Research Board, No. 2029, Transportation Research Board of the National Academies, Washington D.C.

Corthout, R., C.M.J. Tampère, L.H. Immers (2009) Marginal incident computation: an efficient algorithm to determine congestion spillback due to incidents. Proceedings of the Transportation Research Board of the National Academies, Washington, D.C.

D'Este, G.M. and M.A.P. Taylor (2003) Network vulnerability: an approach to reliability analysis at the level of national strategic transport networks. The Network Reliability of Transport: Proceedings of the 1st International Symposium on Transportation Network Reliability (INSTR), Oxford, Pergamon.

Goodwin (1992) A quality margin in transport, in: Traffic Engineering and Control 33 (12), pp. 661-665.

Husdal, J. (2004) Reliability and Vulnerability versus Cost and Benefits. Proceedings of the second International Symposium on Transportation Network Reliability (INSTR),

Christchurch and Queenstown, New Zealand.

Immers, L.H., J.E. Stada, I. Yperman (2004) Robustness and Resilience of Transportation networks; problem survey and examples. Paper presented at NECTAR Cluster Meeting on Reliability of Networks, Amsterdam, March 19-20.

Jamakovic, A., S. Uhlig, I. Theisler (2008) On the relationship between topological metrics in real-world networks, in: Networks and heterogeneous media, Volume 3, Number 2, pp. 345359.

Jonkers, E., J.M. Schrijver, M. Muller (2009) Ietsdoen wellicht niet nodig: tijdverlies als gevolg van vrachtauto-ongevallen door een objectieve bril. Vervoersplanologisch Speurwerk 2009, Antwerpen.

Knoop, V.L., S.P. Hoogendoorn, H.J. van Zuylen (2007) Quantification of the impact of spillback modeling in assessing network reliability. Proceedings of the Transportation Research Board of the National Academies, Washington, D.C.

Knoop, V.L. (2009) Road Incidents and Network Dynamics, Effects on driving behaviour and traffic congestion, T2009/13, December 2009, TRAIL Research School, the Netherlands.

Knoop, V.L., M. Snelder, H.J. van Zuylen, S.P. Hoogendoorn (2010) Link-level Vulnerability Indicators for Real-World Networks, in: Proceedings of the 89th Annual Meeting of the Transportation Research Board, Washington D.C., United States of America, Accepted for publication.

Li, M. (2008) Combining DTA Approaches for Studying Road Network Robustness. Ph.D. Thesis, Trail, Delft University of Technology, The Netherlands. 
Meeuwissen, A.H.M., M. Snelder, J.M. Schrijver (2004) Statistische analyse variabiliteit reistijden voor SMARA. TNO, no. Inro V\&V/2004-31, 04-7N-112-73401, ISBN-number 905986-095-0, 2004, Delft.

Muller, M. (2009) Ex ante evaluatie van de invloed van infrastructuurmaatregelen op de effecten van incidenten. TNO, Delft, The Netherlands.

Murray-Tuite P.M. and H.S. Mahmassani (2004) Methodology for Determining Vulnerable Links in a Transportation Network. Transportation Research Record: Journal of the Transportation Research Board, No.1882, Transportation Research Board of the National Academies, Washington, D.C., pp 88-96.

Nicholson, A., Schmöcker, J, Bell, M.G.H., Iida, Y. (2001) Assessing Transport Reliability: Malevolence and User Knowledge. The Network Reliability of Transport eds M.G.H. Bell and Y. Iida, pp 1-22. Elsevier Science, Kidlington.

Snelder, M., J. Schrijver, R. Landman, J. Mak, M. Minderhoud (2008) De kwetsbaarheid van Randstedelijke vervoernetwerken uit verkeerskundig perspectief. TNO-rapport 2008-DR0882/A, Delft.

Snelder, M. (2009) A Comparison between Dynamec and Indy, TNO - CIRRELT, CIRREL-2009-48.

Tampère M.J., J. Stada, L.H. Immers (2007) Methodology for Identifying Vulnerable Sections in a National Road network. Proceedings of the Transportation Research Board of the National Academies, Washington, D.C.

Taylor, M.A.P. and G.M.D. D'Este (2003) Concepts of network vulnerability and application to the identification of critical elements of transport infrastructure. Paper presented at the 26th Australian Transport Research Forum, Wellington, New Zealand.

Van Lint, J. W. C., Van der Zijpp, N. J. (2003), Improving a travel time estimation algorithm by using dual loop detectors, in: Transportation Research Record: Journal of the Transportation Research Board, 1855, pp. 41-48.

Wakabayashi, H. and Iida, Y. (1992) Upper and lower bounds of terminal reliability of road networks: an efficient method with Boolean algebra. Journal of Natural Disaster Science, 14, $29-44$.

Wilmink, I., A. van den Broeke, L. Berghout (2003) De taakverdeling tussen netwerkstructuur en verkeersmanagement. Vervoersplanologisch Speurwerk 2003: No pay, nо quеие?

Oplossing voor bereikbaarheidsproblemen, Delft.

Yperman, I. (2007) The Link Transmission Model for Dynamic Network Loading. Ph.D. Thesis, Catholic University of Leuven, Belgium. 